\title{
INCREMENTAL PLANAR MOTION
}

\author{
Tony Dean \\ Global Telecom Solutions Sector \\ 1501 West Shure Dr. \\ Motorola, Inc. \\ Arlington Hts., IL 60004, U.S.A.
}

\begin{abstract}
A cellular engineer typically estimates system performance via simulation. An important input to this simulation is the average busy hour subscriber location distribution. The performance of some system features, such as admission control or carrier, antenna, or beam assignment, requires a dynamic mobility model which matches and maintains that distribution. The author discusses the requirements of such a model and presents easily implementable models satisfying those requirements.
\end{abstract}

\section{INTRODUCTION}

A dynamic simulation of a cellular system requires a model of mobility. Simplistic mobility models choose a random velocity vector for a particle (mobile subscriber, automobile, etc.) and compute its destination based on physical laws of motion and the time period, or epoch between simulation steps. This approach does not stabilize the distribution of particle location. More sophisticated approaches model particle motion stochastically, such as (Rose and Yates 1997), (Liu, Bahl, and Chlamtac 1998), (Massey and Whitt 1993), and (Jabbari, Zhou, and Hillier 1998).

In most of these approaches, the object is to assign $a$ priori behavior to the particles in motion, derive the resulting location distribution, and estimate its effects on the systems under study. Our objective is somewhat the opposite. Cellular operators typically have little quantitative information about the behavior of their subscribers, but may have information about their location distribution. A cellular planning tool which incorporates dynamic simulation of subscriber mobility must be able to incorporate this information.

The challenge in this effort is to find a stochastic process that achieves this distribution and satisfies other requirements, which may include

1. Isotropism: Assuming the location distribution information doesn't include directional biases (such as street data), the mobility model should not preferentially choose one direction over another. In other words, in an area of uniform density, the model should be as likely to any one direction as another.

2. Variable velocity: The model must be able to represent a realistic range of different velocities.

3. Variable direction: particles must have access to a realistic range of directions and dynamically change them.

4. Exclusion zones: handsets must be able to avoid exclusion zones and the exterior of the modeled system.

5. Sustained direction: handsets must be able to sustain a direction long enough to produce events, such as handoffs, in a realistic fashion.

In many respects, this is the reversal of the classic stochastic process problem: instead of beginning with the state transition probabilities and solving for the steadystate distribution, one begins with the steady-state distribution and solves for the state transition probabilities subject to the above requirements. The requirements are insufficient to select a single solution, so there is an element of design left in arriving at a solution. This paper addresses one class of solutions.

This problem was previously addressed in (Dean 2000), which presents a mobility model satisfying all requirements except 2 and 3 . The current approach addresses all requirements in a general way. It assumes that motion will be modeled in a plane by a discrete Markov chain. The state space consists of pairs, each corresponding to the current location and orientation of a particle (e.g., a handset). Locations are confined to a rectilinear grid, and motion is affected by movement from one grid location to another. The model approximates motion in directions other than rectilinear by randomly selecting, at each epoch of the chain, from transitions along one of two lateral and vertical directions. The orientation of the particle determines which of the two directions and the probabilities of choice of each. The probabilities are chosen in such a way that the mean angle made with the positive $x$-axis by the vector 
drawn between a starting position of the particle and the following position matches the orientation of the particle

\section{FOUNDATIONS}

\subsection{The State Space}

It is convenient to represent locations on a rectilinear grid as complex numbers. We assume the grid has uniform spacing, so that the complex numbers may be supplied by the set $\mathbb{G}$ of Gaussian integers, i.e. those complex numbers with real and imaginary parts in the set $\mathbb{Z}$ of integers.

Definition 1 A location space is a rectangle $L=\{m+n \mathbf{i} \mid m \in I, n \in J\} \overline{\text { in } \mathbb{G} \text {, where } \mathbf{i}}=\sqrt{-1}$ and I and $J$ are finite intervals in $\mathbb{Z}$. An orientation space is a finite subgroup $\Omega$ of the additive group $\mathbb{R} / 2 \pi \mathbb{Z}$ of angles in radian measure. The set $S=L \times \Omega$ is the state space on which we define Markov chains. Elements of $L$ are called cells and elements of $\Omega$ are called orientations. Given a state $s$ in $S$, the first component is called its cell and the second component is called its orientation. Transitions between states with the same orientation (thus, only the locations are distinct) are translations. Transitions between two states at the same location are rotations.

\subsection{Translations and Rotations}

Our fundamental concept in modeling motion is a planar motion on $S$ in which transitions occur between states $r$ and $s$ only if they have the same orientation and neighboring cells (connected by a unit lateral or vertical line segment) or they share (are in) the same cell. (We formalize this concept in section 2.3.) I.e., the chain moves one step at a time, either one unit vertically or laterally, or makes a change in orientation. Moreover, the orientation of a state limits the cells to which it can make a translation in a special way: If $\mathbf{X}$ is such a chain, let $\mathbf{Z}$ denote the derived chain of cells of $\mathbf{X}$ and let $\mathbf{u}_{n}=\mathbf{Z}_{n+1}-\mathbf{Z}_{n}$, for natural numbers $n \in \mathbb{N}$. We require that the conditional mean of $\mathbf{u}_{n}$, given that $\mathbf{Z}_{n}=\langle z, \theta\rangle$ and the transition $\mathbf{Z}_{n} \rightarrow \mathbf{Z}_{n+1}$ is a translation, makes an angle $\theta$ with the positive real axis. (We will actually be more restrictive than this requirement.) Given these goals, we adopt several conventions:

Definition 2 Let $p$ denote the state transition probability matrix of $\mathbf{X}$. We write the transition probability between orientations $\theta$ and $\varphi$ in the same cell $z$ as $\rho_{z, \theta, \varphi}=$ $p_{\langle z, \theta\rangle,\langle z, \varphi\rangle}$ and the transition probability between neighboring cells (with identical orientation $\theta$ ) as $\tau_{z, w, \theta}=p_{\langle z, \theta\rangle,\langle w, \theta\rangle}$.

Definition 3 If $\boldsymbol{X}$ has a stationary density $f$, then its location space density is $\pi_{z}=\sum_{\theta \in \Omega} f_{z, \theta}$. (We use the boldface, plain symbol " $\pi$ " to denote the number, $\mathrm{pi}$, in order to distinguish it from the probability mass function $\pi$ ).

In any location space, $\pi$ may have cells of zero density, representing exclusion zones where particles cannot reside. Any Markov chain with such a density cannot be irreducible, because there cannot be transitions to or from such cells (i.e., to or from any of the states whose locations are such cells). Moreover, since the Markov chains we will be considering allow only neighbor to neighbor transitions (its "incremental" nature), the support $\underline{L}$ of $\pi$ must be "connected" in the sense that it is the smallest subset closed under the neighbor relation. If $\pi$ has a nonconnected support, we realize $\pi$ as the sum of a finite number of functions each with connected support and apply the techniques herein on normalized versions of each. Since there will be no transitions between the components, the individual solutions will be independent. So, in the sequel, we assume $\underline{L}$ is connected. As a consequence, $\underline{S}=\underline{L} \times \Omega$ is irreducible and, since $\underline{S}$ is finite, $f[\underline{S}$ is unique.

So, to simplify expressions of relationships for states on the "edges" or in exclusion zones of the location space, we assume that $\pi_{z}, p_{\langle z, \theta,\langle z, \varphi\rangle}, \tau_{z, w, \theta}$ and $\tau_{z, \theta}$ (see below) are zero whenever $z$ or $w$ are outside the location space support (including outside $L$ altogether). Furthermore:

Definition 4 We write the probability of a translation out of cell $\mathrm{z}$ in state $s=\langle z, \theta\rangle$ as

$$
\tau_{z, \theta}=\sum_{u^{4}=1} \tau_{z, z+u, \theta}
$$

where the notation " $u=1$ " indicates that $u$ ranges over $\{ \pm 1, \pm \mathbf{i}\}$, thus capturing the probability of translations to any of the neighbors of cell $z$.

\subsection{Incremental Planar Motion}

We formalize the concept of modeling planar motion. As mentioned earlier, we approximate motion in nonrectilinear directions by randomly selecting from between two orthogonal, rectilinear directions. We may represent any two such directions by $u$ and $\mathbf{i} u$, where $u$ is a 4th root of unity (i.e., \pm 1 and $\pm \mathbf{i})$. Suppose $\mathbf{X}$ is a random variable in $\{u, \mathbf{i} u\}$ such that $\mathbb{E}(\mathbf{X})=x+y \mathbf{i}$. By definition, $\mathbb{E}(\mathbf{X})=$ $\mathbb{P}[\mathbf{X}=u] u+\mathbb{P}[\mathbf{X}=\mathbf{i} u] \mathbf{i} u$. Since one of $\{u, \mathbf{i} u\}$ is real and the other imaginary, $|x|=\mathbb{P}[\mathbf{X}= \pm 1]$ and $|y|=\mathbb{P}[\mathbf{X}= \pm \mathbf{i}]$ and $|x|$ $+|y|=1$. If $\theta$ is the angle $\mathbb{E}(\mathbf{X})$ makes with unity,

$$
\begin{aligned}
& \cos \theta=\frac{x}{r}, \quad \sin \theta=\frac{y}{r} \\
& r=\sqrt{x^{2}+y^{2}}
\end{aligned}
$$

Adding absolute values of the equations for $\cos$ and sin together and given that $|x|+|y|=1$, we conclude $r=1 /(|\cos \theta|+|\sin \theta|)$. It is a simple matter to conclude from this that

$$
\begin{array}{r}
\alpha_{\theta} \equiv \operatorname{Pr}[\mathbf{X}= \pm 1]=r|\cos \theta| \\
\beta_{\theta} \equiv \operatorname{Pr}[\mathbf{X}= \pm \mathbf{i}]=r|\sin \theta|
\end{array}
$$


which we take as definitions (the subscripts of $\alpha$ and $\beta$ emphasize their dependency on $\theta$ ). Note that

$$
\sqrt{\alpha_{\theta}^{2}+\beta_{\theta}^{2}}=\sqrt{x^{2}+y^{2}}=|\mathbb{E}(\mathbf{x})| \text {. }
$$

With these definitions in hand, we may define the basic component of modeling planar motion.

Definition 5 A planar motion is a Markov chain $\mathbf{X}=\langle\mathbf{Z}, \Theta\rangle$ on $S$ such that

1. If $\pi$ is the stationary density of $\mathbf{X}$, it is uniform on the orientations states in any cell; i.e., for each $z \in$ $\mathrm{L}$ and $\theta, \varphi \in \Omega$, we have $\pi_{\langle\mathrm{z}, \theta\rangle}=\pi_{\langle\mathrm{z}, \varphi\rangle}$.

2. Transitions consist only of translations and rotations.

We call $\mathbf{X}$ an incremental planar motion if it exhibits the additional properties:

3. Translations occur only between neighboring cells; i.e., the transition probabilities $\left\langle p_{s, t}: s, t \in S\right\rangle$ are such that if $s=\langle z, \theta\rangle$ and $s=\langle w, \theta\rangle$, then $p_{s, t}>0$ implies $|z-w| \leq 1$.

4. If $\tau_{z, \theta}$ is as defined in equation (1), then for each 4-th root $u$ of unity,

$$
\tau_{z, z+u \theta}=\left\{\begin{array}{ll}
\alpha_{\theta}, & \operatorname{Re}(u) \cos \theta>0 \\
\beta_{\theta}, & \operatorname{Im}(u) \sin \theta>0 \\
0, & \text { otherwise }
\end{array}\right\} .
$$

For each $z \in L$, we write $\pi_{z}=\sum_{\theta \in \Omega} \pi_{z, \theta}$. Because of Property $1, \pi_{z, \theta}=\pi_{z} / \mid \Omega$ for each $\theta$ in $\Omega$. In the sequel, references to the stationary probability density of a planar motion refer actually to the density of the location space. For convenience, we say that the matrix $p$ with such properties is planar incremental. Property 4 requires that in orientation $\theta$, translations occur only in the directions of the real or imaginary components of $e^{\mathrm{i} \theta}$, and with probabilities $\alpha_{\theta}$ and $\beta_{\theta}$. The earlier discussion shows that the mean translation, given orientation $\theta$, is $x+y \mathbf{i}$.

Because an incremental planar motion may move only one unit at a time along the real or imaginary axes, it is useful to define symbols which represent those units for any orientation state $\theta$. We define $u_{\theta}$ and $v_{\theta}$, in parallel with $\alpha_{\theta}$ and $\beta_{\theta}$, as the real and imaginary 4 th roots of unity which lie along the real and imaginary parts (if non-zero) of $e^{\mathrm{i} \theta}$ : $u_{\theta}=\operatorname{sgn}(\cos \theta), v_{\theta}=\mathbf{i} \operatorname{sgn}(\sin \theta)$, where $\operatorname{sign}(t)$ is $+1,-1$, or 0 depending on whether $t$ is positive, negative, or zero.

\subsection{A Classification Scheme for Probability Flows}

The key to understanding incremental planar motions lies in classifying their transition probabilities. For Markov chains, the Chapman-Kolmogorov equations express the idea that the probability of a given state is equal to the flow of probability from all other states into the given state. The flow of probability from a state $r$ to a state $s$ is $\pi_{r} p_{r, s}$. Because of the nature of an incremental planar motion, its flows are either from one cell to an adjacent cell with identical orientation or from one orientation to another within a cell. The following definitions classify these flows.

Let $\pi$ denote the stationary density of the incremental planar motion $\mathbf{Z}$ on state space $S$. We define the translational flows egres and ingress as follows.

Definition 6 Given $s=\langle z, \theta\rangle \in S$, the egress from $s$ is the sum $T_{E}(s)$ of all flows from $s$ to states with neighboring cells and identical orientation. Similarly, the ingress towards $s$ is the sum $T_{I}(s)$ of all flows from states with neighboring cells and identical orientation. Formally, they are

$$
\begin{aligned}
& T_{E}(z, \theta)=\sum_{u^{4}=1} \pi_{z, \theta} p_{z, z+u ; \theta}=\pi_{z, \theta} \tau_{z, \theta} \\
& T_{I}(z, \theta)=\sum_{u^{4}=1} \pi_{z-u, \theta} p_{z-u, z ; \theta}
\end{aligned}
$$

In a like manner, we define the rotational flows divergence and convergence:

Definition $7 \quad$ The divergence from $s$ is the sum $R_{E}(s)$ of all flows from $s$ to states with identical cells (but with the same or different orientations). The convergence with $s$ is the sum $R_{I}(s)$ of all flows into s from states with identical cells. Formally,

$$
\begin{aligned}
& R_{E}(z, \theta)=\sum_{\varphi \in \Omega} \pi_{\langle z, \theta\rangle} \rho_{z, \theta, \varphi}=\pi_{\langle z, \theta\rangle} \sum_{\varphi \in \Omega} \rho_{z, \theta, \varphi} . \\
& R_{I}(z, \theta)=\sum_{\varphi \in \Omega} \pi_{\langle z, \varphi\rangle} \rho_{z, \varphi, \theta}
\end{aligned} .
$$

\section{EXPLICIT MODELS OF INCREMENTAL PLANAR MOTION}

We construct an explicit class of incremental planar motions. Later, we incorporate motions in this class into useful models of planar motion. Suppose that the functions $\tau . L \times \Omega \rightarrow[0,1]$ and $\rho: L \times \Omega \times \Omega \rightarrow[0,1]$ are defined such that for each $z \in L$ and $\theta \in \Omega, \tau_{z, \theta}+\sum_{\varphi \in \Omega} \rho_{z, \theta, \varphi}=1$. Then, we say

that $\tau$ and $\rho$ describe an incremental planar motion. We extend these functions to a matrix $p$ of transition probabilities on the state space $S=L \times \Omega$ by defining

$$
\begin{aligned}
& p_{\langle z, \theta\rangle,\left\langle z+u_{\theta}, \theta\right\rangle}=\alpha_{\theta} \tau_{z, \theta} \text { for } u_{\theta} \neq 0 \\
& p_{\langle z, \theta\rangle,\left\langle z+v_{\theta}, \theta\right\rangle}=\beta_{\theta} \tau_{z, \theta} \text { for } v_{\theta} \neq 0 \\
& p_{\langle z, \theta\rangle,\langle z, \phi\rangle}=\rho_{z, \theta, \phi}
\end{aligned}
$$


and $p_{\langle z, \theta,\langle w, \phi\rangle}=0$, otherwise. We refer to $p$ as the incremental planar matrix characterized by $\tau$ and $\rho$. By definition, $p$ is the transition probability matrix for an incremental planar motion.

The following result shows, given $\pi$ and a definition for $\tau$, how to construct a definition for $\rho$ that characterizes a transition probability matrix $p$ with stationary distribution $\pi$.

Theorem 1. Suppose $\pi$ is a probability mass function on the location space $L$ in the state space $S=L \times \Omega$. Then (there are two parts):

1. Suppose $\tau$ and $\rho$ describe an incremental planar motion on $S=L \times \Omega$ and $p$ is the incremental planar matrix characterized by them. If $\pi$ is a stationary distribution for $p$ then for each cell $z \in L$, the total egress is equal to the total ingress. I.e.,

$$
\sum_{\theta \in \Omega} T_{E}(z, \theta)=\sum_{\theta \in \Omega} T_{I}(z, \theta) .
$$

2. Suppose only $\tau$ (but not $\rho$ ) is given, but we know that the total egress equals the total ingress (i.e., $\tau$ and $\pi$ satisfy the conclusion of Part 1). Then for each $z$, $\theta$ and $\varphi$, we may define

$\rho_{z, \theta, \varphi}=$

$$
\frac{\left(\pi_{z}-T_{E}(z, \theta)\right)\left(\pi_{z}-T_{I}(z, \varphi)\right)}{\pi_{z} \sum_{\psi \in \Omega}\left(\pi_{z}-T_{E}(z, \psi)\right)}
$$

if $\sum_{\psi \in \Omega}\left(\pi_{z}-T_{E}(z, \psi)\right)>0$ and $p_{z, \theta, \phi}=0$ otherwise. Then $\tau$ and $\rho$ describe an incremental planar motion and $\pi$ is the stationary distribution for the incremental planar matrix $p$ characterized by $\tau$ and $\rho$.

proof: Part 1 . We appeal to the principle that the flow of probability out of a state is equal to the flow into the state. Given our previous definitions, this amounts to $T_{E}(z, \theta)+R_{E}(z, \theta)=T_{I}(z, \theta)+R_{I}(z, \theta)$. If we sum these equations over all $\theta$ in $\Omega$, the contributions from $R_{E}$ and $R_{I}$ are

$$
\begin{aligned}
& \sum_{\theta \in \Omega} R_{E}(z, \theta)=\sum_{\theta \in \Omega} \sum_{\varphi \in \Omega} \pi_{z} \rho_{z, \theta, \varphi} \\
& \sum_{\theta \in \Omega} R_{I}(z, \theta)=\sum_{\theta \in \Omega} \sum_{\varphi \in \Omega} \pi_{z} \rho_{z, \varphi, \theta}
\end{aligned} .
$$

These sums are equal, since the roles of $\theta$ and $\phi$ can be interchanged. Thus, these terms cancel out, leaving $\sum_{\theta \in \Omega} T_{E}(z, \theta)=\sum_{\theta \in \Omega} T_{I}(z, \theta)$, which is the conclusion.
Part 2. First, we must show that for each $z$ and $\theta$, the conditional probabilities conditioned on $\langle z, \theta\rangle$ sum to 1 . If the denominator in the expression for $\rho_{z, \theta, \varphi}$ is zero, it is because $\tau_{z, \theta}=1$ for every orientation state $\theta$ at location $z$, so this is trivial. Otherwise,

$$
\begin{aligned}
& \sum_{\varphi \in \Omega} \rho_{z, \theta, \varphi}=\frac{\left(\pi_{z}-T_{E}(z, \theta)\right)}{\pi_{z} \sum_{\psi \in \Omega}\left(\pi_{z}-T_{E}(z, \psi)\right)} \sum_{\varphi \in \Omega}\left(\pi_{z}-T_{I}(z, \varphi)\right) \\
& =\frac{\pi_{z}-T_{E}(z, \theta)}{\pi_{z}} \frac{N_{\Omega} \pi_{z}-\sum_{\varphi \in \Omega} T_{I}(z, \varphi)}{N_{\Omega} \pi_{z}-\sum_{\psi \in \Omega} T_{E}(z, \psi)}
\end{aligned}
$$

By hypothesis, the numerator and denominator of the second fraction are equal. Thus,

$$
\sum_{\varphi \in \Omega} \rho_{z, \theta, \varphi}=\left(\pi_{z}-T_{E}(z, \theta)\right) / \pi_{z} .
$$

Since $T_{E}(z, \theta)=\pi_{z} \tau_{z, \theta}$, rearranging terms and dividing by $\pi_{z}$ shows that $\tau_{z, \theta}+\sum_{\varphi \in \Omega} \rho_{z, \theta, \varphi}=1$. This is equivalent to the statement that the sum of all conditional probabilities is 1 , since $\tau_{z, \theta}=\tau_{z, z+u, \theta}+\tau_{z, z+v, \theta}$, where $u=u_{\theta}$ and $v=v_{\theta}$.

Finally, we must show that the Chapman-Kolmogorov equations are satisfied. This is similar to the previous argument, but we sum over $\theta$ instead of $\varphi$ :

$$
\begin{aligned}
& \sum_{\theta \in \Omega} \rho_{z, \theta, \varphi}=\frac{\left(\pi_{z}-T_{I}(z, \varphi)\right)}{\pi_{z} \sum_{\psi \in \Omega}\left(\pi_{z}-T_{E}(z, \psi)\right)} \sum_{\theta \in \Omega}\left(\pi_{z}-T_{E}(z, \theta)\right) \\
& =\frac{\pi_{z}-T_{I}(z, \varphi)}{\pi_{z}}
\end{aligned}
$$

Since $T_{I}(z, \varphi)=\pi_{z-u} \tau_{z-u, \theta}+\pi_{z-u} \tau_{z-v, \theta}$, where $u=u_{\theta}$ and $v=v_{\theta}$, we may rearrange the above equation into the form of the Chapman-Kolmogorov equations. If the denominator is zero, so that $\rho_{z, \theta, \varphi}=0$ instead, the hypothesis implies that $\sum_{\varphi \in \Omega}\left(\pi_{z}-T_{I}(z, \varphi)\right)=0$ as well. Since these are non-negative terms, we conclude that $\pi_{z}-T_{I}(z, \theta)=0$. Since $\rho_{z, \theta, \varphi}=0$ for all $\varphi$, we arrive at the Chapman-Kolmogorov equations anyway. (QED)

\subsection{Canonical Incremental Planar Motion}

By means of Theorem 1, we define an explicit class of models of incremental planar motion. Let $\lambda_{\theta} \in(0,1)$ for each $\theta \in \Omega$ and for each $z \in L$, let

$$
\tau_{z, \theta}= \begin{cases}\left(\lambda_{\theta} / \pi_{z}\right) \min \left(\pi\left[Q_{\theta}(z)\right]\right), & \pi_{z}>0 \\ 0, & \pi_{z}=0\end{cases}
$$


where $\pi[A]=\left\{\pi_{a} \mid a \in A\right\}$ and

$Q_{\theta}=\left\{z, z+u_{\theta}, z+v_{\theta}, z+u_{\theta}+v_{\theta}\right\}$, the directional quadrant of

$z$. (Note that our convention that $\pi_{w}=0$ for $w$ not in $L$ implies that this formula works even for edge cells.) So, for $\pi_{z}>0, \tau_{z, \theta}$ is $\lambda / \pi_{z}$ times the smallest density in the quadrant containing $z, z+u_{\theta}$, and $z+v_{\theta}$. Under suitable conditions described in the next theorem, $\tau$ and $\rho$ (as defined in Theorem 1, Part 2) lead to what we call a canonical model of incremental planar motion.

Theorem 2. Assume that $\pi \in \Omega$, so that the orientation group is "symmetric" about both lateral and vertical axes. Suppose $\pi$ is a probability mass function on $L, \tau$ is defined as in equation (2), $\rho$ is defined as in Theorem 1 , Part 2, and for each $\theta$ in $\Omega, \lambda_{\theta}=\lambda_{\pi-\theta}=\lambda_{-\theta}$ (i.e., $\lambda$ is the same regardless of reflection through either axis). Then $\tau$ and $\rho$ describe an incremental planar motion and $\pi$ is the stationary distribution for the incremental planar matrix $p$ characterized by $\tau$ and $\rho$.

proof: We show that the hypotheses of Theorem 1, Part 2 are satisfied. The ingress at cell $z$ is

$$
\sum_{\theta \in \Omega} \alpha_{\theta} \tau_{z-u_{\theta}, \theta} \pi_{z-u_{\theta}}+\beta_{\theta} \tau_{z-v_{\theta}, \theta} \pi_{z-v_{\theta}} .
$$

Note that $\tau_{z, \theta} \pi_{z}=\lambda_{\theta} \min \left\{\pi_{z+u} \mid u=0, u_{\theta}, v_{\theta}, u_{\theta}+v_{\theta}\right\}$. The mappings $\theta \rightarrow \pi-\theta$ and $\theta \rightarrow-\theta$ amount to reflection through the $y$ - and $x$-axes respectively (angular arithmetic is modulo 2 $2 \pi$ ). Thus, $\pi_{z-u} \tau_{z-u, \theta}=\pi_{z} \tau_{z, \pi-\theta}$ and $\pi_{z-\nu} \tau_{z-v, \theta}=\pi_{z} \tau_{z,-\theta}$, where $u=u_{\theta}$ and $v=v_{\theta}$. Substituting these relationships into the expression for ingress yields

$$
\sum_{\theta \in \Omega} \alpha_{\theta} \tau_{z, \pi-\theta} \pi_{z}+\beta_{\theta} \tau_{z,-\theta} \pi_{z} .
$$

Since $\alpha_{\theta}$ and $\beta_{\theta}$ are unchanged by reflection through either $x$ - or $y$-axis, the above simplifies to

$$
\sum_{\theta \in \Omega} \alpha_{\pi-\theta} \tau_{z, \pi-\theta} \pi_{z}+\beta_{-\theta} \tau_{z,-\theta} \pi_{z}
$$

By distributing the sum over both terms and individually re-indexing the resulting sums, we arrive at the expression for egress. (QED)

\section{MOTION SCHEMAS}

The model described in the previous section may be generalized beyond the location space distribution, since there are specific formulas specifying how the model should be constructed for any location space distribution.

Definition $8 \quad$ For a fixed $\Omega$, we define an incremental planar motion schema as a mapping from pairs $\langle S, \pi\rangle$ of state spaces and probability mass functions to pairs of functions $\langle\tau(\mathrm{S}), \rho(\mathrm{S})\rangle$ such that

1. $\tau(\pi): L \times \Omega \rightarrow[0,1]$ and $\rho(\pi): L \times \Omega \times \Omega \rightarrow[0,1]$ describe an incremental planar motion on $L \times \Omega$ with mass density $\pi$.

2. Given $\left\langle S^{\prime}, \pi^{\prime}\right\rangle$ such that $S=L \times \Omega, L \subseteq L^{\prime}$ and $\pi$ is the conditional density of $\pi^{\prime}$ given $L$, then $\pi\left(\pi^{\prime}\right)$ and $\rho\left(\pi^{\dagger}\right)$ are extensions of $\pi(\pi)$ and $\rho(\pi)$.

A planar motion $\mathbf{X}$ on the state space $S$ with stationary density $\pi$ whose state transition matrix is characterized by $\tau(\pi)$ and $\rho(\pi)$ is an instance of the schema.

The exact nature of the formalism is important only to the extent that it captures the nature of an incremental planar motion independently of the distribution $\pi$. In a typical simulation, there is a single state space with a fixed distribution, yet distinct particles will be assigned different incremental motion schemas. The behavior of the particle is then a function of its schema and the distribution of the location space in which it resides. Innate properties of the schema will express themselves differently in different location spaces. One important such property is the mean speed of the schema, as we describe in the next section.

The typical location space mass density function consists of a few broad regions of uniform density. For that reason, it is convenient to describe properties of motion schemas in terms of their behavior in a location space of uniform density. By convention, if a behavior of a planar motion is measurable in some sense, we assign to a motion schema the limiting value of that measure in all instances of the schema on uniformly distributed location spaces as the location space increases without limit.

\section{PARTICLE VELOCITY}

Let $\mathbf{X}$ be an incremental planar motion, and let $\mathbf{Z}$ and $\Theta$ be the chains which represent the locations and orientations at each step of $\mathbf{X}$; i.e., for each $n, \mathbf{X}_{n}=\left\langle\mathbf{Z}_{n}, \boldsymbol{\Theta}_{n}\right\rangle$. Given a state $s=\left\langle z_{n}, \theta_{n}\right\rangle$, the instantaneous velocity of $\mathbf{X}$ at $s$ is $V_{z, \theta}=\mathbb{E}\left(\mathbf{Z}_{1}-\mathbf{Z}_{0} \mid \mathbf{X}_{0}=\langle z, \theta\rangle\right)$ and its mean instantaneous speed in state $s$ is $\left|V_{z, \theta}\right|$, where we take the width and height of a cell as unit distance and one epoch as unit time. Note that $V_{z, \theta}=\alpha_{\theta} \tau_{z, \theta} u_{\theta}+\beta_{\theta} \tau_{z, \theta} v_{\theta}$ and the instantaneous speed is $\left|V_{z, \theta}\right|=\tau_{z, \theta} \sqrt{\alpha_{\theta}^{2}+\beta_{\theta}^{2}}$.

In general, the instantaneous speed is dependent on the distribution $\pi$. For a canonical incremental planar motion, the instantaneous speed is

$$
\left|V_{z, \theta}\right|=\sqrt{\alpha_{\theta}^{2}+\beta_{\theta}^{2}} \frac{\lambda_{\theta}}{\pi_{z}} \min \pi\left[Q_{\theta}(z)\right]
$$


when $\pi_{z}>0$, and zero otherwise.

For a fixed $\theta$, the mean speed is higher where neighboring cells in the direction of $\theta$ have higher densities relative to $z$, while the mean speed is lower where these densities are lower relative to $z$. Thus, a particle moves more rapidly towards areas of increasing density than towards those of decreasing density. In this way, the distribution $\pi$ impedes or enhances the mean instantaneous speed of the particle. However, if the particle is in a region $R$ of uniform density and $Q_{\theta}(z) \subseteq R$, the instantaneous speed is given by $\left|V_{z, \theta}\right|=\lambda_{\theta} \sqrt{\alpha_{\theta}^{2}+\beta_{\theta}^{2}}$. This expression is independent of $\pi$ and independent of $z$ away from any edge. So, following the convention set forth in Section 4, we refer to it as the speed of the schema in orientation $\theta$.

\section{MEAN RUN LENGTH}

In simulations of wireless mobility, it is important to be able to maintain the orientation of motion of a handset, so that it produces handoff events in a realistic fashion. Let $\Theta$ denote the chain of orientations of an incremental planar motion. If $\mathbf{M}=\min \left(m \geq 0 \mid \boldsymbol{\Theta}_{m+1} \neq \boldsymbol{\Theta}_{0}\right)$, then the mean run length $l_{R}(z, \theta)$ from $z$ in orientation $\theta$ is $l_{R}(z, \theta)=\left|\mathbb{E}\left(\mathbf{Z}_{\mathbf{M}}-z \mid \mathbf{Z}_{0}=z, \boldsymbol{\Theta}_{0}=\theta_{0}\right)\right|$. For motion schemas, we define $l_{R}(z, \theta)$ as the maximum of this value over all state spaces with uniform location space densities. The quantity $l_{R}(z, \theta)$ is then a measure of the mean run length of a particle if it were unimpeded by density or location space boundaries.

The quantity $l_{R}(z, \theta)$ is difficult to compute in the general case. However, the following (stated without proof) is true for incremental planar motion schemas:

Theorem 3. If $\langle\tau, \rho\rangle$ is a canonical incremental planar motion schema, then

$$
l_{R}(z, \theta)=\frac{\lambda_{\theta}}{1-\lambda_{\theta}-\rho_{\theta, \theta}} \sqrt{\alpha_{\theta}^{2}+\beta_{\theta}^{2}} .
$$

\section{ISOTROPY}

If the motion we wish to model has no a priori bias in direction at a particular location, any motion schema which models such motion should be "isotropic"; i.e., the instantaneous speed in any direction at that location should be independent of direction. A reasonable interpretation of "no bias" is that all the cells in the neighborhood $N(z)=\{z+a+b \mathbf{i}: a, b=0, \pm 1\}$ of a given cell $z$ have the same probability. To be precise, an incremental planar motion schema $\langle\tau, \rho\rangle$ is isotropic if for each state space $S=L \times \Omega$, if $z \in \Omega$ and $N(z)$ is unbiased, then $\left|V_{z, \theta}\right|$ is identical for all $\theta$.

For a canonical incremental planar motion schema if $z \in L$ and $N(z)$ is unbiased, then $\tau_{z, \theta}=\lambda_{\theta}$ for all $\theta$. If velocity is to be independent of orientation, $\left|V_{z, \theta}\right|=\lambda_{\theta} \sqrt{\alpha_{\theta}^{2}+\beta_{\theta}^{2}}$ must be a constant $C$, so $\lambda_{\theta}=C / \sqrt{\alpha_{\theta}^{2}+\beta_{\theta}^{2}}$. Since $\lambda_{\theta} \leq 1$, $C \leq 1 / \sqrt{2}=\min \left\{\sqrt{\alpha_{\theta}^{2}+\beta_{\theta}^{2}} \mid \theta \in \Omega\right\}$. Thus, we conclude that

$$
\lambda_{\theta}=\frac{\mu}{\sqrt{2} \sqrt{\alpha_{\theta}^{2}+\beta_{\theta}^{2}}}=\frac{|\cos \theta|+|\sin \theta|}{\sqrt{2}} \mu
$$

where $\mu$ is a constant $\leq 1$, which we call the motility. We call a canonical incremental planar motion so equipped an isotropic canonical incremental planar motion. Substituting $\lambda_{\theta}$ for $\tau_{z, \theta}$ in the expressions for $T_{E}$ and $T_{I}$ yields $T_{E}(z, \theta)=\pi_{z} \lambda_{\theta}$ and $T_{I}(z, \theta)=\pi_{z} \lambda_{\theta}$, so by the definition in Theorem 1, Part 2,

$$
\rho_{z, \theta, \varphi}=\frac{\left(1-\lambda_{\theta}\right)\left(1-\lambda_{\varphi}\right)}{\sum_{\psi \in \Omega}\left(1-\lambda_{\psi}\right)} .
$$

\section{PRACTICAL MODELS OF PLANAR MOTION}

In a discrete event simulation, we may choose the epoch of a canonical incremental planar motion schema to whatever value is needed to model any target particle velocity. However, if the simulation has a common fixed epoch size, we must be more inventive.

In such cases, one can speed up a particle's motion by an integral factor of $n$ by scaling its "epoch" to a fraction $1 / n$ of the main epoch. It is not difficult to see that the resulting motion at each "main" epoch is still Markov with the same distribution. But suppose we have a canonical incremental planar motion $\mathbf{X}$ with motility $\mu$ and we wish to simulate a particle with instantaneous speed of $v$ units per epoch, where $v$ is a possibly non-integral positive real number. If $v>\mu$, we can get close with this technique by taking $n=\lceil v / \mu\rceil$. However, a more accurate approach is to construct an incremental planar motion $\mathbf{X}^{\prime}$ from $\mathbf{X}$ whose mean speed is $v / n<\mu$, then achieve the incremental planar motion $\mathbf{Y}$ with mean speed $v$ by "speeding up" $\mathbf{X}^{\prime}$ by a factor of $n$. The next theorem, stated without proof, shows how $\mathbf{X}^{\prime}$ is derived from $\mathbf{X}$.

Theorem 4. Let $\mathbf{X}$ be an isotropic incremental planar motion and let $\mathbf{B}$ be a chain of independent, identically distributed Bernoulli variables independent of $\mathbf{X}$. Define $\mathbf{N}(k)=\sum_{i<k} \mathbf{B}_{i}$ for $\mathrm{k} \in \mathbb{N}$ and let $\mathbf{Y}=\mathbf{X}_{\mathbf{N}}$. Then

1. $\quad \boldsymbol{Y}$ is an isotropic incremental planar motion with the same stationary distribution $\pi$ as $\boldsymbol{X}$.

2. If the translations and rotations of $\mathbf{X}$ are $\left\langle\tau_{z, \theta}\right|$ $z \in L, \theta \in \Omega\rangle$ and $\left\langle\rho_{\theta, \varphi} \mid \theta, \varphi \in \Omega\right\rangle$, then the transla- 
tions and rotations of $\mathbf{Y}$ are $\left\langle\tau_{z, \theta}^{\prime} \mid z \in L, \theta \in \Omega\right\rangle$ and $\left\langle\rho_{\theta, \varphi}^{\prime} \mid \theta, \varphi \in \Omega\right\rangle$, where

$$
\begin{aligned}
& \tau_{z, \theta}^{\prime}=\lambda \tau_{z, \theta} \\
& \rho_{z, \theta, \varphi}^{\prime}=\left\{\begin{array}{cc}
1-\lambda\left(1-\rho_{z, \theta \theta \theta}\right), & \varphi=\theta . \\
\lambda \rho_{z, \theta, \varphi}, & \varphi \neq \theta
\end{array}\right.
\end{aligned}
$$

3. If $\lambda=\operatorname{Pr}\left[\mathbf{B}_{0}=1\right]$ and $v$ is the instantaneous speed of $\mathbf{X}$, then the mean instantaneous speed of $\mathbf{Y}$ is $\lambda v$.

\section{CONCLUSIONS}

In the Introduction, we present a set of requirements for simulating particle motion particularly suited to wireless mobile applications. In particular these requirements include that the model match an a priori particle location mass density function and that the mean duration of particle orientation is controllable. We satisfy these requirements with a class of particle motion models called $c a$ nonical incremental planar motions. We then show that by judicious choice of the epoch between movements, these models may be extended to model motion at whatever speeds are required for simulation.

A requirement that remains to be addressed is location dependent speed. In many applications mean particle speed has a location dependent aspect, and a model of mobility should include it. (E.g., in wireles cellular applications, subscriber speed may be lower in high density areas and higher in low density areas.) One way that planar incremental motion might be expanded to model location dependent speed is to add another dimension $V$ to the state space: $S=L \times \Omega \times V$, where $V$ is a finite set of speeds. If the mass density $\pi$ is extended to this space and $\langle z, \theta\rangle$ is a fixed point in $L \times \Omega$, then $\pi_{z, \theta}(v)=\pi(z, \theta, v)$ is a distribution of speeds in that state. There are some choices that must be made in this approach. If the incremental nature of the motion is worth keeping, then as a particle passes from one region to another, it could only transition to a new speed that is a "neighbor" of the one it already has. Thus, the particle speed would evolve toward the mean speed of the region rather than make a sharp transition to it. Although such behavior models a kind of acceleration, which is at least superficially realistic, it remains to be seen if it could be controlled in a more essentially realistic manner or if it is useful at all. The other option would be to abandon the incremental nature of speed transition, but this would significantly expand challenges to finding simple models.

\section{REFERENCES}

Dean, T. 2000. A Method For Achieving Stable Distributions Of Wireless Mobile Location In Motion Simulations. In Proceedings of the 2000 Winter Simulations
Conference, ed. J. A. Joines, R. R. Barton, K. Kang, and P. A. Fishwick, 456-463. Piscataway, New Jersey: Institute of Electrical and Electronics Engineers.

Jabbari, B., Y. Zhou, and F. Hillier. 1998. Random walk modeling of mobility in wireless networks. Proceedings of the 1998 Vehicular Technology Conference: 639-643

Liu, T., P. Bahl, and I. Chlamtac. 1998. Mobility Modeling, location tracking, and trajectory prediction in wireless ATM networks. IEEE journal on selected areas in communications 16 (6): 922-936.

Massey, W. and W. Whitt. 1993. Networks of infiniteserver queues with nonstationary Poisson input. Queueing Networks 13: 183-250.

Rose, C. and R. Yates. 1997. Location uncertainty in mobile networks: a theoretical framework. IEEE Coтmunications Magazine 35 (2) : 94-101.

\section{AUTHOR BIOGRAPHY}

TONY DEAN is a distinguished member of technical staff at Motorola, Inc., where he conducts performance analysis and modeling of cellular infrastructure systems. His research interests lie in the discrete event simulation of such systems. Prior to joining Motorola, he contributed in the development of a patented token-ring based voice and data communications system, engaged in software development for factory data collection terminals, and supported software products at Digital Equipment Corp. and Burroughs Corp. (now Unisys). He holds a Ph.D. degree in mathematical logic from Indiana University. His email address is $<$ Anthony.Dean@motorola.com>. 